\title{
Interference Mitigation Techniques Using Receiver Processing and Resource Allocation
}

\author{
Jesvin Veancy Boaz', Yogesh Palanichamy² \\ ${ }^{1}$ Department of Electronics and Communication Engineering, Easwari Engineering College, Chennai, India \\ ${ }^{2}$ Department of Information Science and Technology, Anna University, Chennai, India \\ Email: jesvinveancy.b@srmeaswari.ac.in, yogesh@annauniv.edu
}

Received 29 April 2016; accepted 23 May 2016; published 5 August 2016

Copyright (C) 2016 by authors and Scientific Research Publishing Inc.

This work is licensed under the Creative Commons Attribution International License (CC BY). http://creativecommons.org/licenses/by/4.0/

(c) (i) Open Access

\begin{abstract}
Heterogeneous network consists of the pico cells overlaid over the macro cell coverage area in a wireless cellular network. The pico cells are deployed to increase the capacity of the homogeneous network by reusing the spectrum further. However, more users will tend to be associated to the macro cell due to the fact that the transmit power of the pico cell is low. In order to increase the number of users associated to the pico cell, range extension techniques like biased association are used. This will cause severe interference to cell edge users of the pico cell from the macro cell causing degradation in throughput performance in the cell range extension area. In this paper, interference mitigation using receiver processing along with different scheduling techniques is proposed to improve the throughput, average delay, and the packet delivery ratio performance of the system. The performance comparison of the round robin, proportional fair and modified largest weighted delay first (MLWDF) algorithm for resource allocation using interference suppressing receiver is done, and analyzed. It is shown that the MLWDF algorithm achieves the highest throughput with minimum average delay of packets with the best delivery ratio.
\end{abstract}

\section{Keywords}

Cellular Network, Orthogonal Frequency Division Multiplexing, Interference, Scheduling, Post Processing Signal to Noise Ratio

\section{Introduction}

The mobile broadband traffic is growing exponentially during the recent years due to the evolution of the capa- 
bilities of the terminals [1]. The mobile service providers are facing severe challenges to meet the growing demand of the very high data rate applications. Spectrum is a scarce resource and various techniques are required to increase the network capacity and meet the growing needs of data rate [2]. Adding more antennas to enhance the capacity will increase the signal processing between the transmitter and receiver. Shrinking of the macro cell size in urban and dense areas by deploying more base stations a.k.a. eNodeBs (eNBs) by operators is also done to increase the capacity [1]-[6]. This will increase the interference to the system. The problem of this method is that it is very expensive to add more eNBs due to the need for expensive drive tests in finding suitable locations for deployment, and also the high real estate cost to put the towers. The alternative approach is to introduce low power and less planned pico eNBs overlaid on the macro network. This is called as heterogeneous networks (HetNet) [1] [4]. The pico nodes are added depending on the local capacity needs [2]. However, the interference due to addition of the pico nodes is more severe due to the fact that the macro and pico transmit at different power. An example of a HetNet that consists of one pico eNB overlaid on a macro eNB coverage area is shown in Figure 1.

The coverage of the pico eNB is quite limited due to its low transmit power, and the strong interference that user equipment (UE) receives from the macro eNB. In this scenario, the equal signal to interference plus noise (SINR) boundary where the downlink (DL) SINR observed from the macro eNB and the pico eNB is the same is very closer to the pico cell. Therefore, only a small percentage of UEs are associated to the pico eNB [6]. Due to lack of sufficient number of UEs, the spectrum is not fully utilized by the pico eNBs. Therefore, new techniques are required to increase the network efficiency of HetNets by offloading macro cell traffic to pico cells [6]. The performance of the network is significantly improved when more UEs are allowed to attach to the pico eNB by further reusing the frequency resources enabling additional cell-splitting gains [2]. More UEs are associated to the pico eNB by applying a positive bias to the SINR observed with respect to the pico eNB, and the UEs that are thus associated to the pico eNB are called as cell range extension or expansion (CRE) UEs, and the region is called as CRE region [2]. However, biasing makes the interference further worse for the UEs served by the pico eNB at the boundary between the macro and pico cells. Therefore, efficient interference mitigation techniques are required to achieve the real benefits of adding the low power picos [2]-[4] [7]. The heterogeneous network that illustrates the CRE region is shown in Figure 2.

A single technique cannot fully mitigate the interference, and therefore, more than one techniques are required to mitigate the effect of interference in a cellular system. In this paper, one such interference mitigation technique based on receiver processing along with resource allocation using appropriated scheduling method is proposed to improve the throughput, average packet delay, and packet delivery ratio performance of the system. The performance is compared and analyzed with the round robin (RR) [8]-[10], proportional fair (PF) [9] [10], and modified largest weighted delay first (MLWDF) [10] algorithm along with interference suppression receiver to decide on resource allocation. It is found from simulation results that the MLWDF algorithm achieves

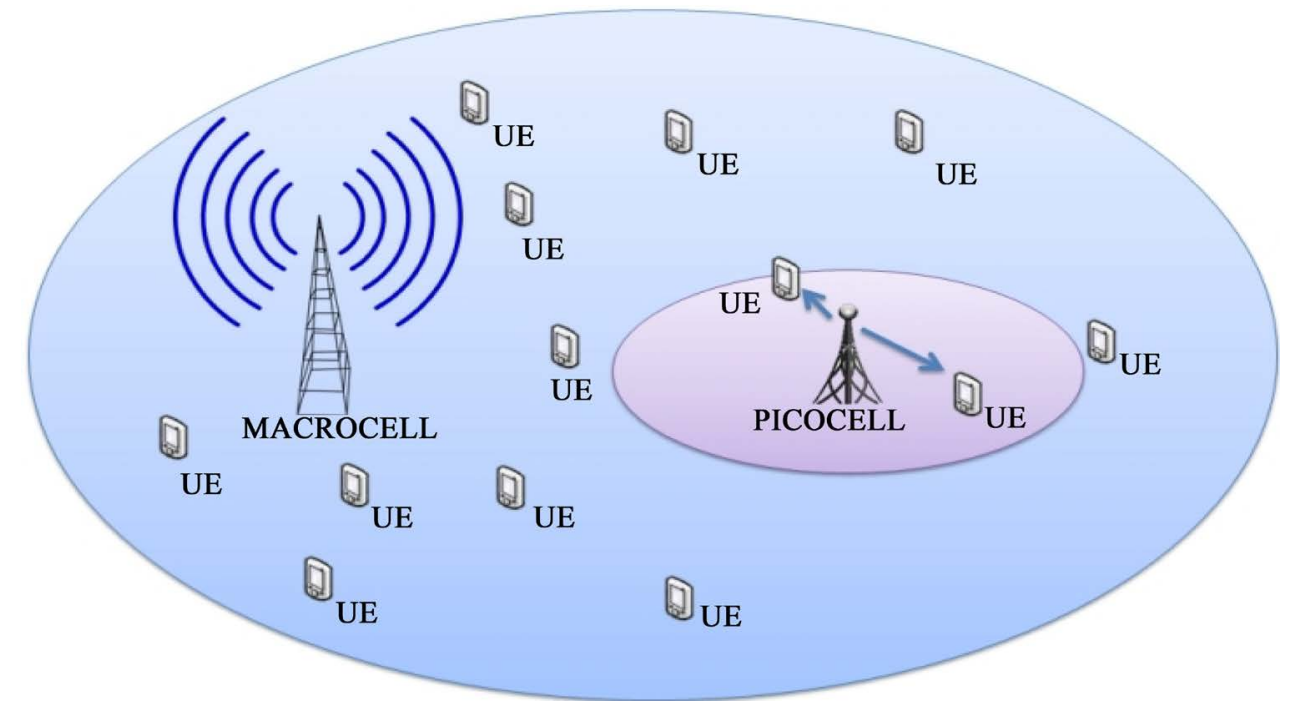

Figure 1. Example of a HetNet with one macro and a pico eNB. 


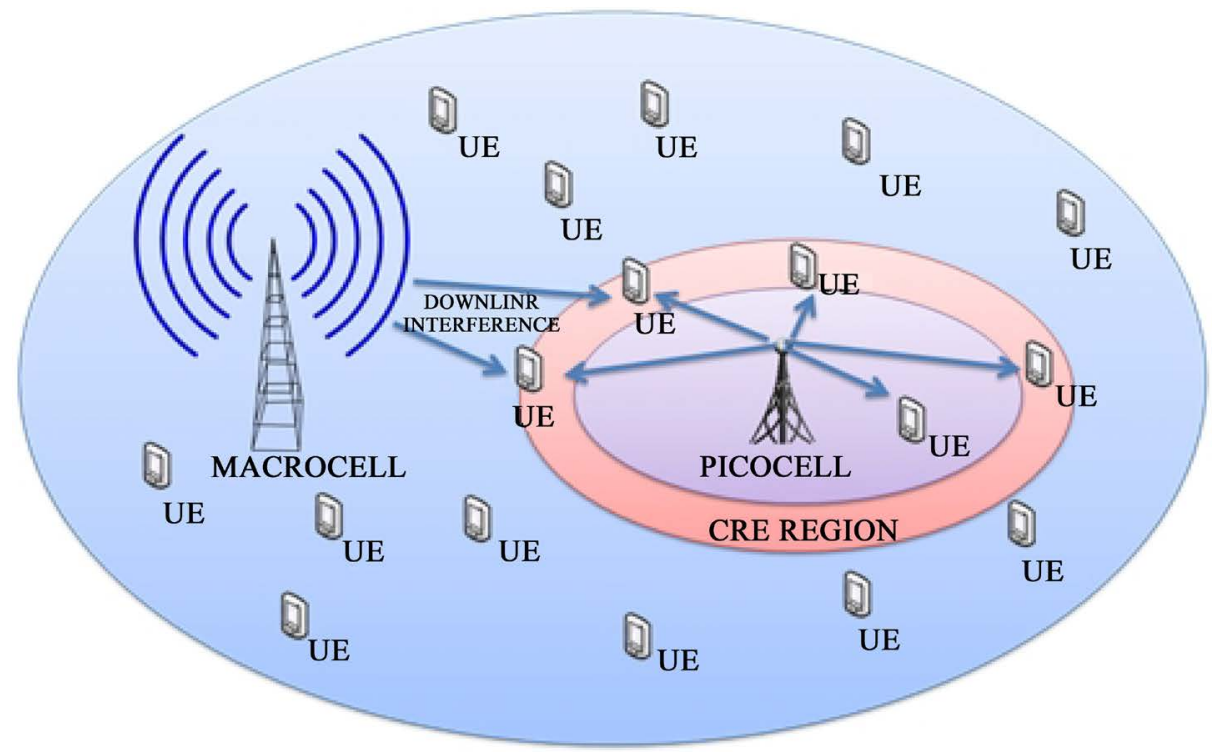

Figure 2. Downlink interference from macro and range extension.

the highest throughput with minimum average delay of packets with the best delivery ratio compared to the PF and RR schedulers.

\section{System Model}

The fourth generation (4G) long term evolution (LTE) technology is considered to simulate the proposed interference mitigation methods. The DL transmission of LTE is based on orthogonal frequency division multiplexing access (OFDMA) technique for providing services to multiple UEs. OFDMA divides the total available bandwidth into multiple parallel narrow band carriers called subcarriers. The resource to be allocated for data transmission consists of 12 such subcarriers over six or seven OFDM symbols a.k.a resource block (RB). The numbers of RBs are 25, 50, and 100 for the bandwidth of 5, 10, and $20 \mathrm{MHz}$, respectively. The UEs depending on the data rate requirement will be allocated with a RB or group of RBs [3] [4] [9] [11] [12].

The system consists of $L$ eNBs, each having $N_{t}$ antennas, and $Q$ UEs, each having $N_{r}$ antennas. The total transmit power of the desired eNB is $P_{s}$ and interfering eNB is $P_{i}, i=1,2, \cdots, L-1$, Therefore, the $N_{r} \times 1$ vector $\boldsymbol{r}(\boldsymbol{n}, \boldsymbol{k})$ received by the $N_{r}$ antennas at the UE for the $N_{t} \times 1$ vector $\boldsymbol{s}(\boldsymbol{n}, \boldsymbol{k})$ transmitted by the $N_{t}$ antennas at the eNB is given by the following equation

$$
\boldsymbol{r}(\boldsymbol{n}, \boldsymbol{k})=\sqrt{P_{s}} \boldsymbol{H}(\boldsymbol{n}, \boldsymbol{k}) \boldsymbol{s}(\boldsymbol{n}, \boldsymbol{k})+\sum_{i=1}^{L-1} \sqrt{P_{i}} \boldsymbol{I}_{i}(\boldsymbol{n}, \boldsymbol{k}) \boldsymbol{u}_{i}(\boldsymbol{n}, \boldsymbol{k})+\boldsymbol{v}(\boldsymbol{n}, \boldsymbol{k}) .
$$

where, $\boldsymbol{H}(\boldsymbol{n}, \boldsymbol{k})$ and $\boldsymbol{I}_{i}(\boldsymbol{n}, \boldsymbol{k})$ are the $N_{r} \times N_{t}$ desired and interference channel matrix from the ith eNB, respectively on the subcarrier $\boldsymbol{k}$ at time instant $\boldsymbol{n}$ in the frequency domain, and they are given by

$$
\boldsymbol{H}(\boldsymbol{n}, \boldsymbol{k})=\left[\begin{array}{cccc}
h_{11} & h_{12} & \cdots & h_{1 N_{t}} \\
h_{21} & h_{22} & \cdots & h_{2 N_{t}} \\
\vdots & \vdots & \ddots & \vdots \\
h_{N_{r} 1} & h_{N_{r} 1} & \cdots & h_{N_{r} N_{t}}
\end{array}\right], \quad I_{i}(\boldsymbol{n}, \boldsymbol{k})=\left[\begin{array}{cccc}
i_{11} & i_{12} & \cdots & i_{1 N_{t}} \\
i_{21} & i_{22} & \cdots & i_{2 N_{t}} \\
\vdots & \vdots & \ddots & \vdots \\
i_{N_{r} 1} & i_{N_{r} 1} & \cdots & i_{N_{r} N_{t}}
\end{array}\right] .
$$

The entries $h_{j m}$ of $\boldsymbol{H}(\boldsymbol{n}, \boldsymbol{k})$ and $i_{j m}$ of $\boldsymbol{I}_{i}(\boldsymbol{n}, \boldsymbol{k})$ are the coefficients of desired and interference channels, respectively from the $m$ th antenna at eNB to the $j$ th antenna at UE. They are obtained using complex Gaussian random variable with zero mean and unit variance for each tap of a frequency selective multipath channel. The variance of each tap is appropriately scaled based on the 3GPP defined power delay profile [13], and the frequency domain channel is obtained using fast Fourier transform (FFT). The $N_{t} \times 1$ vector $\boldsymbol{u}_{i}(\boldsymbol{n}, \boldsymbol{k})$ is the interfering signal transmitted from the ith eNB. The entries of the $N_{r} \times 1$ noise vector $\boldsymbol{v}(\boldsymbol{n}, \boldsymbol{k})$ are obtained using complex Gaussian random variable with zero mean and $N_{0}$ variance. Then, the preprocessing SINR is 
given by

$$
\operatorname{SINR}_{\text {pre }}=\frac{P_{s}}{N_{0}+\sum_{i=1}^{L-1} P_{i}} .
$$

The signal to interference ratio (SIR) for a system with $L$ eNBs is defined as

$$
S I R=10 \log \left(\frac{P_{s}}{\sum_{i=1}^{L-1} P_{i}}\right) .
$$

The signal to noise ratio (SNR) for various SIR is defined as $\mathrm{SNR}=P_{s} / N_{0}$.

The SNR is varied by varying the noise variance $N_{0}$ for link level simulations.

\section{Interference Mitigation Techniques}

The extra antennas at the receiver could be used to cancel or suppress the interference at the receiver. It is well known that a system with $N_{r}$ receive antennas can cancel up to $N_{r}-1$ interferers [7]. Maximum likelihood (ML) receivers are used to exactly cancel the interferers [14] with full knowledge of the desired and all the interference channels. They are computationally complex as it requires to jointly decoding the desired and interference data. However, there is another class of receivers, which may not be able to fully cancel the interference, but can suppress the effect of interference to a minimum, and are called as minimum mean square error (MMSE) receivers [15]-[17]. This is a less complex receiver as it requires knowing only the interference covariance, and not the exact interfering channels. The performance of these receivers is enhanced with a slight increase in complexity when some of interfering channels are known, and are called as eMMSE receivers [16] [17].

\subsection{MMSE Receiver}

The receiver operation is performed on every subcarrier at each time instant in the OFDM system. Therefore, without loss of generality, the time and frequency indices can be dropped from (1), and considering $P_{s}=P_{i}=1$ the received signal equation is written as

$$
\boldsymbol{r}=\boldsymbol{H} \boldsymbol{s}+\sum_{i=1}^{L-1} \mathbf{I}_{i} \mathbf{u}_{i}+\boldsymbol{v}
$$

It can be rewritten as

$$
\boldsymbol{r}=\boldsymbol{H} \boldsymbol{s}+\boldsymbol{w} .
$$

where, $\boldsymbol{w}$ is the total noise plus interference vector of size $N_{r} \times 1$. The low complexity receiver weights for the MMSE solution is given by

$$
\boldsymbol{B}_{M M S E}=\boldsymbol{H}^{\boldsymbol{H}} \boldsymbol{R}_{M M S E}^{-1} .
$$

where, $\boldsymbol{R}_{\text {MMSE }}$ is the total received signal covariance matrix estimate, and is given by

$$
\boldsymbol{R}_{M M S E}=\boldsymbol{H} \boldsymbol{H}^{H}+\boldsymbol{R}_{w w} .
$$

where, $\boldsymbol{R}_{w w}$ is the covariance matrix of inter-cell interference and white noise, and is given by

$$
\boldsymbol{R}_{w w}=w w^{H} .
$$

Therefore, the estimate of $s$ is given by

$$
\hat{\boldsymbol{s}}=\boldsymbol{B}_{\text {MMSE }} \boldsymbol{r} .
$$

\section{2. eMMSE Receiver}

When one of the dominant interference is known, (4) can be written as

$$
\boldsymbol{r}=\boldsymbol{H} \boldsymbol{s}+\boldsymbol{I}_{1} \boldsymbol{u}_{1}+\sum_{i=2}^{L-1} \boldsymbol{I}_{i} \boldsymbol{u}_{i}+\boldsymbol{v} .
$$

It can be rewritten as 


$$
\boldsymbol{r}=\boldsymbol{H} \boldsymbol{s}+\boldsymbol{I}_{1} \mathbf{u}_{1}+\boldsymbol{x} .
$$

where, $\boldsymbol{X}$ is sum of the noise plus rest of the interference vector of size $N_{r} \times 1$. The low complexity receiver weights for the MMSE solution is given by

$$
\boldsymbol{B}_{\text {eMMSE }}=\boldsymbol{H}^{\boldsymbol{H}} \boldsymbol{R}_{\text {eMMSE }}^{-1} \text {. }
$$

where, $\boldsymbol{R}_{\text {eMMSE }}$ is the total received signal covariance matrix estimate, and is given by

$$
\boldsymbol{R}_{e M M S E}=\boldsymbol{H} \boldsymbol{H}^{\boldsymbol{H}}+\boldsymbol{I}_{1} \boldsymbol{I}_{1}^{\boldsymbol{H}}+\boldsymbol{R}_{x x} .
$$

where, $\boldsymbol{R}_{x x}$ is the covariance matrix of noise plus rest of the interference, and is given by

$$
R_{x x}=x x^{H} .
$$

Therefore, the estimate of $s$ is given by

$$
\hat{\boldsymbol{s}}=\boldsymbol{B}_{\text {eMMSE }} \boldsymbol{r} .
$$

The simulation results in this paper are obtained for the MMSE and eMMSE receiver by performing Monte Carlo simulations of the link and system level simulation assuming the channel state information (CSI) $\boldsymbol{H}$ known only at the UE for various antenna configurations. Also, for MMSE receiver the noise plus interference covariance $\boldsymbol{R}_{w w}$, and for eMMSE receiver one dominant interference $\boldsymbol{I}_{1}$ and noise plus rest of the interference covariance $\boldsymbol{R}_{w w}$ are assumed to be known. These assumptions will only help decouple the channel estimation problem, and will not in any way affect the conclusion drawn in this paper. Moreover, eMMSE receiver is used to obtain the post processing SINR [12] [14] [15] for making scheduling decisions for system simulations.

Figure 3 shows the link level performance comparison of MMSE and eMMSE receivers with two eNBs having one transmit antenna each, and a UE for two and three antennas. The UE is assumed to be located at equidistance from both eNBs so that the SIR is $0 \mathrm{~dB}$, which reflects the worst case interference from one eNB when the desired and interfering eNB transmits at the same power. As expected, the eMMSE receiver is able to cancel the interference fully with knowledge of the one interfering channel for two UE antennas whereas the MMSE is flooring after $15 \mathrm{~dB}$. Note that with three antennas MMSE receiver also suppress the interference completely and get an array gain of $3 \mathrm{~dB}$ when compared to the two antenna eMMSE receiver. However, with three UE antennas, the eMMSE receiver is also able to achieve diversity gain using the extra antenna, which is evident from the change in slope of the curve.

Figure 4 shows the link level performance comparison of MMSE and eMMSE receivers with three eNBs having one transmit antenna each, and a UE for two, three and four antennas. The UE is assumed to be located

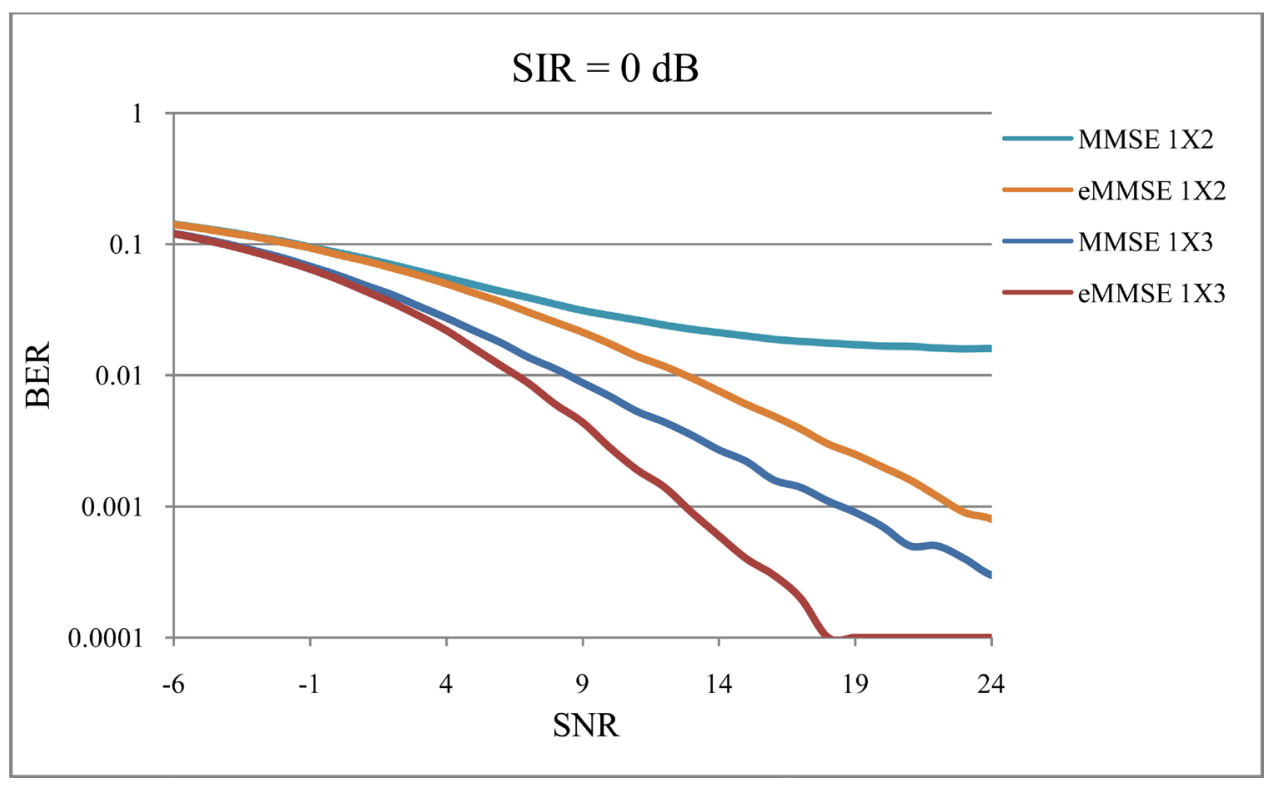

Figure 3. BER performance comparison of MMSE and eMMSE receiver with one dominant interferer. 


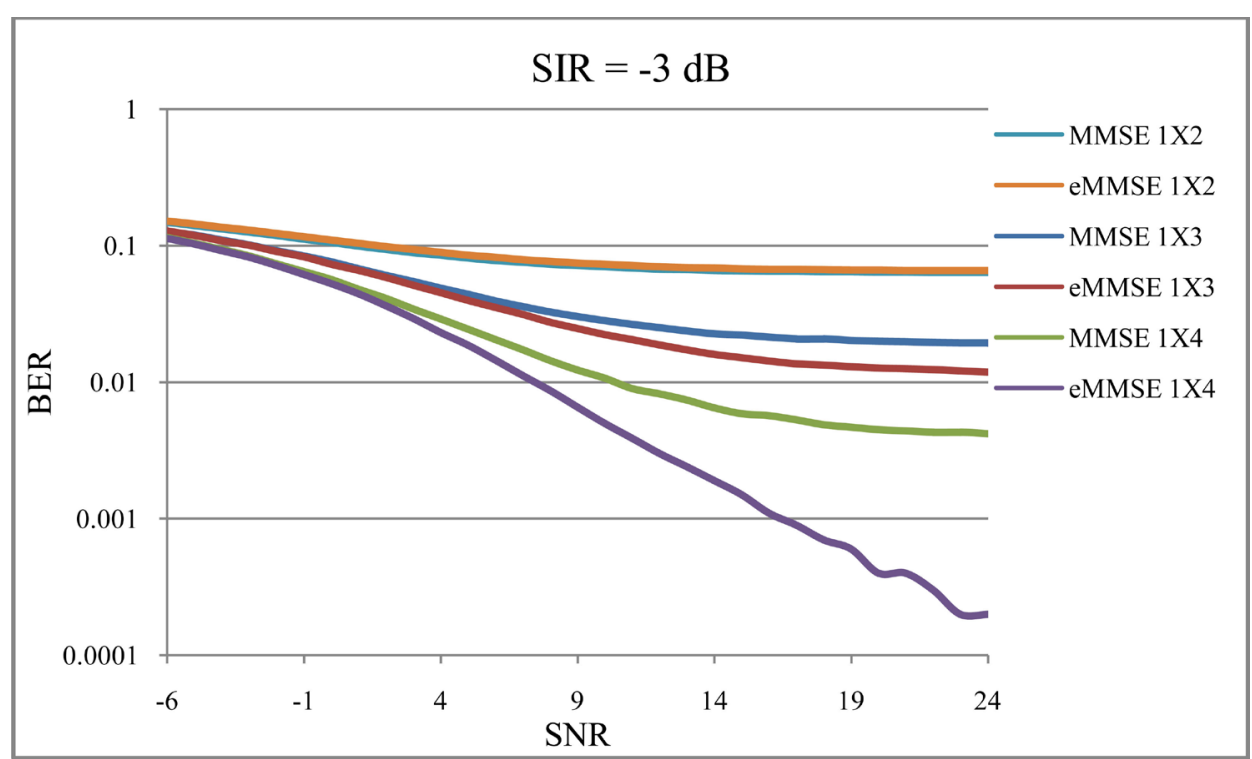

Figure 4. BER performance comparison of MMSE and eMMSE receiver with two dominant interferers.

at equidistance from all the three eNBs so that the SIR is $-3 \mathrm{~dB}$, which reflects the worst case interference from two eNB when the desired and interfering eNBs transmit at the same power. It is observed that both receivers are not able to suppress the interference fully with two and three antennas at the UE as it is evident due to the flooring after $15 \mathrm{~dB}$. This is because either no or one interference channel is assumed to be known, and there are two dominant interferences. Note that with increase in number of UE antennas, interference suppression gain is increased for both the receivers. However, with four UE antennas, the eMMSE receiver is able to suppress the interference completely and achieve diversity gain using the extra antenna as evident from the slope of the curve.

\section{Scheduling}

The radio resource management (RRM) functions including packet scheduling are performed by the eNB in LTE. The scheduling is performed for a frequency resource corresponding to a time period of $1 \mathrm{~ms}$, and is called as the transmit time interval (TTI) [11]. The number of UEs that can be scheduled in any given TTI is limited by the number of RBs in the frequency band and the scheduler [8] [10].

The functionalities of an eNB relevant to packet scheduling are illustrated in Figure 5. A buffer to store the incoming packets is assigned at the eNB for each UE. These packets that are arriving into the buffer are queued for transmission to the respective UEs. The arrival time of the packets are time stamped, and are taken out of the buffer for transmission on a first-in-first-out (FIFO) basis.

The UE computes the post processing SINR at the MMSE or eMMSE receiver, which are obtained from (4) to (7), respectively, and are given as

$$
\operatorname{SINR}_{\text {post }}^{M M S E}=\left[\boldsymbol{H}^{\boldsymbol{H}}\left(\boldsymbol{H} \boldsymbol{H}^{\boldsymbol{H}}+\boldsymbol{R}_{w w}\right)^{-1} \boldsymbol{H}\right] / N_{0},
$$

and

$$
\operatorname{SINR}_{\text {post }}^{\text {eMMSE }}=\left[\boldsymbol{H}^{\boldsymbol{H}}\left(\boldsymbol{H} \boldsymbol{H}^{\boldsymbol{H}}+\boldsymbol{I}_{1} \boldsymbol{I}_{1}^{\boldsymbol{H}}+\boldsymbol{R}_{x x}\right)^{-1} \boldsymbol{H}\right] / N_{0},
$$

The post processing SINR is obtained using either (8) or (9) on every resource element (subcarrier) in the RB, and is converted in to a single effective SINR using mean mutual information bit (MMIB) [18] for a given bit error rate (BER). This value is mapped to a channel quality indicator (CQI), and is reported back to the eNB. The CQI is used by the eNB to choose appropriate modulation and coding scheme (MCS) to transmit the information bits in the physical channel [9]. This is called as link adaptation and a.k.a adaptive modulation and coding (AMC). The RBs with high post processing SINR is an indication of less interference in those RBs. When 


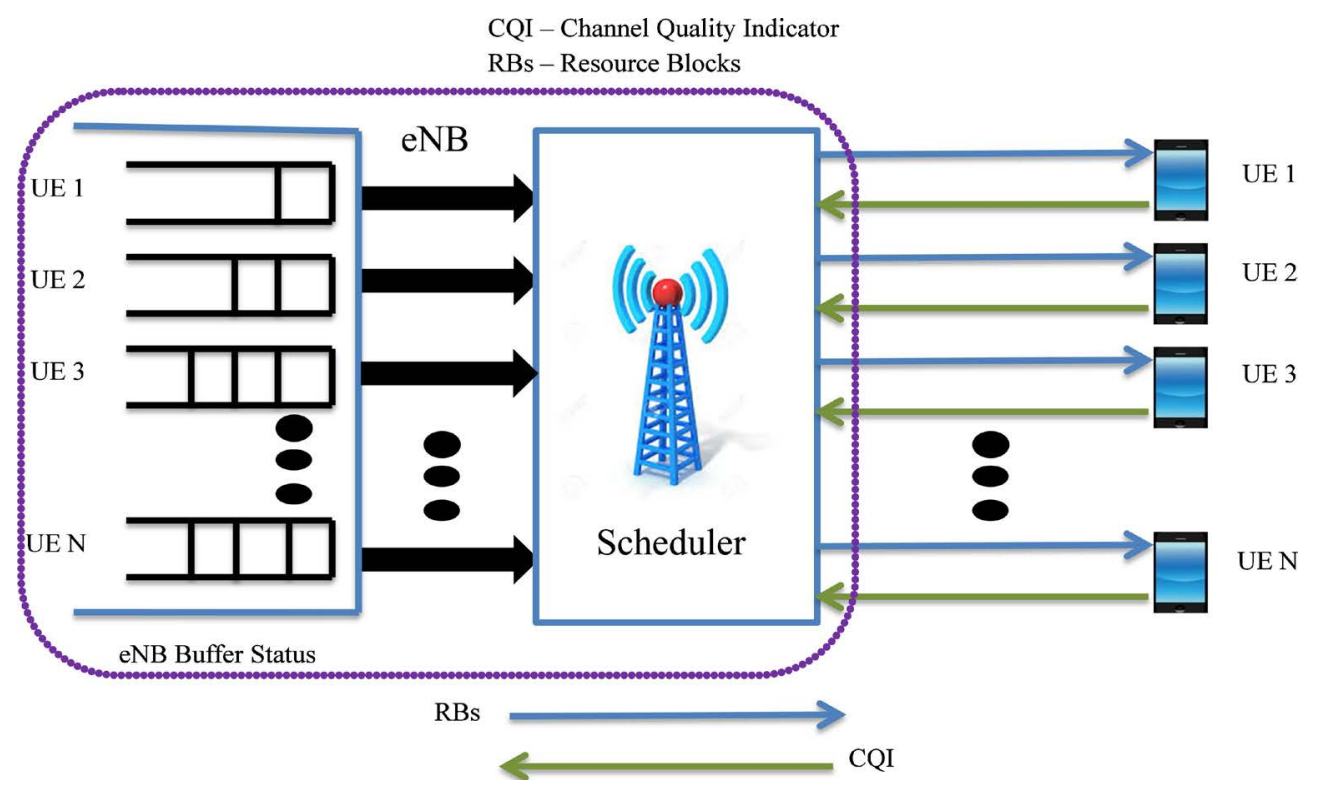

Figure 5. Communication system to illustrate the scheduling functionalities.

this is used as a metric for scheduling at the eNB, it will ensure maximum interference suppression at the allocated resources when either of the MMSE or eMMSE receivers is used at the UE for data detection.

As the DL of LTE uses OFDMA as the multiple access scheme, it allows the eNB to perform opportunistic scheduling along both time and frequency to ensure that the allocations are given to an UE with the best post processing SINR in a particular portion of the band at a given point of time. The packet scheduler determines the UE to be scheduled on a RB based on its reported CQI, and other fairness or QoS metrics for PF and MLWDF schedulers, except in the case of RR scheduler that is independent of the channel conditions.

\subsection{Round Robin Scheduler}

The RR scheduler is simple resource allocation method that equally distributes the resources to all UEs [8]-[10]. The resources are allocated to available UEs in a cyclic fashion irrespective of their channel conditions. Hence, the overall system throughput will be low due to the possibility of channel conditions being poor at the time of actual resource allocation. The resource allocation can be done either in time domain, or frequency domain, or both of the time frequency resource in OFDMA systems. The RR scheduler is easy to implement, and it will provide the best fairness in resource allocation to UEs, however, at the cost of throughput.

\subsection{Proportional Fair Scheduler}

The PF scheduler aims at improving throughput without severely affecting the fairness by allocating resources to the UEs whenever their channel conditions are good with respect to their own average throughput of the past [9] [10]. It provides a trade-off between throughput and fairness, and is best suited for multiuser systems like OFDMA where the resources are shared in both time and frequency domain to exploit the multiuser diversity in the system. The PF scheduler is very suitable to support non real time traffic (NRT) traffic types as it ensures fairness among UEs over a long period of time. The metric to be computed on every RBs in each sub frame for all UEs to be scheduled is given by

$$
P_{m 1}=\left(\frac{r_{q}(t)}{R_{q}(t)}\right) .
$$

where,

$r_{q}(t)$ is the instantaneous achievable data rate for a UE in a particular RB by its respective CQI obtained from the post processing SINR of the receiver computed using (8) or (9), and

$R_{q}(t)$ is the average data rate of the $q$ th UE at time $t$ obtained by averaging the data rate supported by this 
UE in the past over a long period of time.

The PF algorithm decides the UEs to be scheduled on a particular RB based on the priority function given below

$$
P_{f}=\underset{u_{q}}{\arg \max }\left(P_{m 1}\right)=\underset{u_{q}}{\arg \max }\left(\frac{r_{q}(t)}{R_{q}(t)}\right) .
$$

where, $u_{q}$ is the delay threshold of the $q$ th UE.

\subsection{Modified Largest Weighted Delay First Scheduler}

The MLWDF scheduler aims to support real time traffic with additional performance metrics [10]. MLWDF when compared to the PF scheduler introduced additional two parameters, i.e. the head of line (HoL) packet delay and quality of service (QoS). For each packet in the queue, HoL packet delay, which is the time difference between the current time and the arrival time of the packet, is computed. Different delay deadlines are assigned to packets according to the QoS requirements for various services. The scheduler assigns RBs to different flows by considering the properties of the traditional PF rule and the HoL packet delay for the real time flows. Packets belonging to a real time service will be discarded when the target delay deadline assigned to that UE is exceeded while waiting at the MAC buffer. Therefore, the modified PF rule is appropriately weighted, and is given by

$$
P_{m 2}=W_{q}(t)\left(\frac{r_{q}(t)}{R_{q}(t)}\right) .
$$

where,

$r_{q}(t)$ is the instantaneous achievable data rate for a UE in a particular RB by its respective CQI obtained from the post processing SINR of the receiver computed using (8) or (9),

$R_{q}(t)$ is the average data rate of the $q$ th UE at time $t$ obtained by averaging the data rate supported by this UE in the past over a long period of time, and

$W_{q}(t)$ is the HoL packet delay of the $q$ th UE at time $t$.

The MLWDF algorithm decides the UEs to be scheduled on a particular RB based when the delay exceeds its threshold $t_{q}$ is decided based on the following priority function

$$
P_{M}=\underset{a_{q}}{\arg \max }\left(P_{m 2}\right)=\underset{a_{q}}{\arg \max } W_{q}(t)\left(\frac{r_{q}(t)}{R_{q}(t)}\right) .
$$

where,

$$
a_{q}=-\left(\frac{\log p_{q}}{t_{q}}\right),
$$

$t_{q}$ is the delay threshold of the $q$ th UE, and

$p_{q}$ denotes the probability of HoL delay to exceed its threshold for the qth UE $a_{q}$.

\section{Simulation Results}

The system level simulations to compare the performance of RR, PF, and MLWDF scheduler are performed using the network simulator tool NS2 for LTE-Advanced (LTE-A) system considering $5 \mathrm{MHz}$ bandwidth with 25 RBs. The scheduling is done on a sub frame basis of $1 \mathrm{~ms}$ TTI as per LTE/LTE-A technologies and in one TTI all 25 RBs are scheduled to different UEs using one of the RR, PF, and MLWDF schedulers. After the users are scheduled for one TTI, the throughput is calculated as sum of all information bits transmitted successfully over all resource elements, which is obtained as the product of the number of bits in the modulation scheme with the code rate and $(1-B E R)$.

A cell of radius 500 meters consisting of a macro eNB at the centre, and two pico eNBs are placed in the center of a non overlapping random circular pico cell of radius 40 metres. A total of 40 UEs are considered with 24 UEs dropped uniformly in the entire cell, and 8 UEs each are dropped in the pico cell. The macro and pico eNBs transmit with a transmit power of $43 \mathrm{dBm}$ and $30 \mathrm{dBm}$, respectively. Due to the difference in transmit power 
between the macro and the pico eNBs, more UEs tend to attach to the macro due to high SINR. To balance the load between the macro and the pico eNB, biasing is applied. i.e., a bias value of $0,6,9$, and $15 \mathrm{~dB}$ is added to the preprocessing SINR given by (3). The UEs are attached to the strongest eNB depending on the modified preprocessing SINR after adding the bias value. Simulations are performed for multiple realizations by changing the bias values using RR, PF, and MLWDF schedulers for resource allocations. The post processing SINR is computed using (9) for all three schedulers in rate and throughput calculations, and applied in the priority function (11) and (13) for PF and MLWDF schedulers.

The performance of various schedulers in LTE system using interference mitigation receiver is obtained using system level simulations with the setup explained above. The throughput performance comparison of the various scheduling algorithm for different bias value is shown in Figure 6. It is evident from the figure that the throughput improves with the increase in bias values for all the three schedulers. This is due to the fact that the UEs are able to get more resources from moving to the pico eNB, and also thereby releasing more resources to the UEs attached to the macro eNB. The performance of RR scheduler is poor compared to the other two schedulers as it does not take into account of the CQI for scheduling. However, the MLWDF and PF scheduler perform better as it schedules UE with best channel and less interference in a particular resource block at a given time instant. Moreover, the best throughput performance is achieved by the MLWDF algorithm due to the fact that the fairness is determined by the QoS and delay permitted. This shows that it is advantageous to use MLWDF algorithm in real time systems.

The comparison of the average delay with increase in bias values for the PF, MLWDF and RR scheduling is shown in Figure 7. The increase in average delay with increase in the bias value is due to the fact that biasing results in increase in the interference when the UEs are moved from macro to pico eNB. This will reduce the post processing SINR, and hence less number of bits is transmitted in the allocated resources. It will in turn increase the delay in sending and receiving the packets. Here again the RR scheduler performs poor as the UEs are allocated with resources irrespective of the channel and interference conditions. However, the PF and MLWDF scheduler utilize the channel condition and the interference suppression capability of the receiver well, and hence can send more bits in their allocated resources, and hence reduce the average delay. It is found that the average delay is the least in the MLWDF algorithm, and this is due to the fact that QoS and delay metrics are used over PF during resource allocation.

The comparison of the packet delivery ratio with increase in bias values for the PF, MLWDF and RR scheduler is shown in Figure 8. The increase in the packet delivery ratio with increase in bias value is due to the fact that the UEs are able to get more resources from moving to the pico eNB, and also thereby releasing more resources to the UEs attached to the macro eNB. Moreover, the delivery ratio will increase with post processing SINR and the choice of the performance metric. It is evident that the MLWDF scheduler has the best perfor-

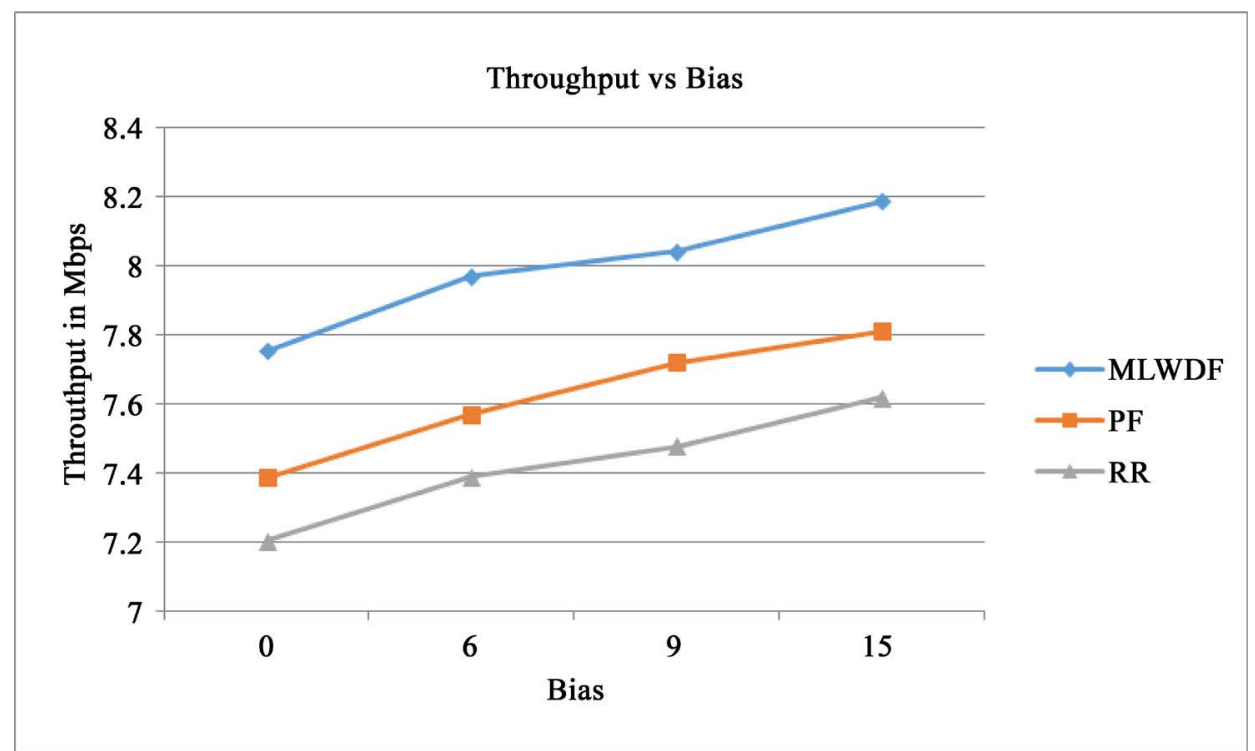

Figure 6. Throughput performance comparison for various bias values using MLWDF, PF and RR schedulers. 


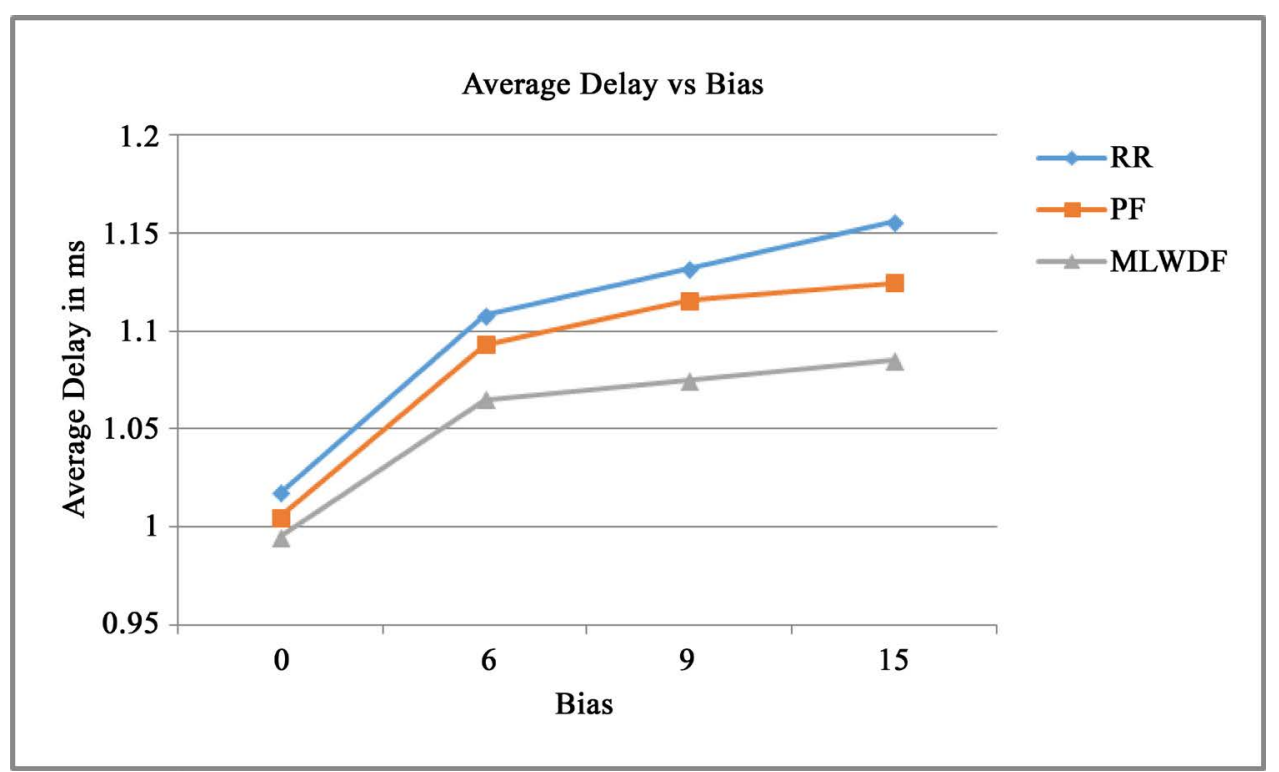

Figure 7. Average delay performance comparison for various bias values using MLWDF, PF and RR schedulers.

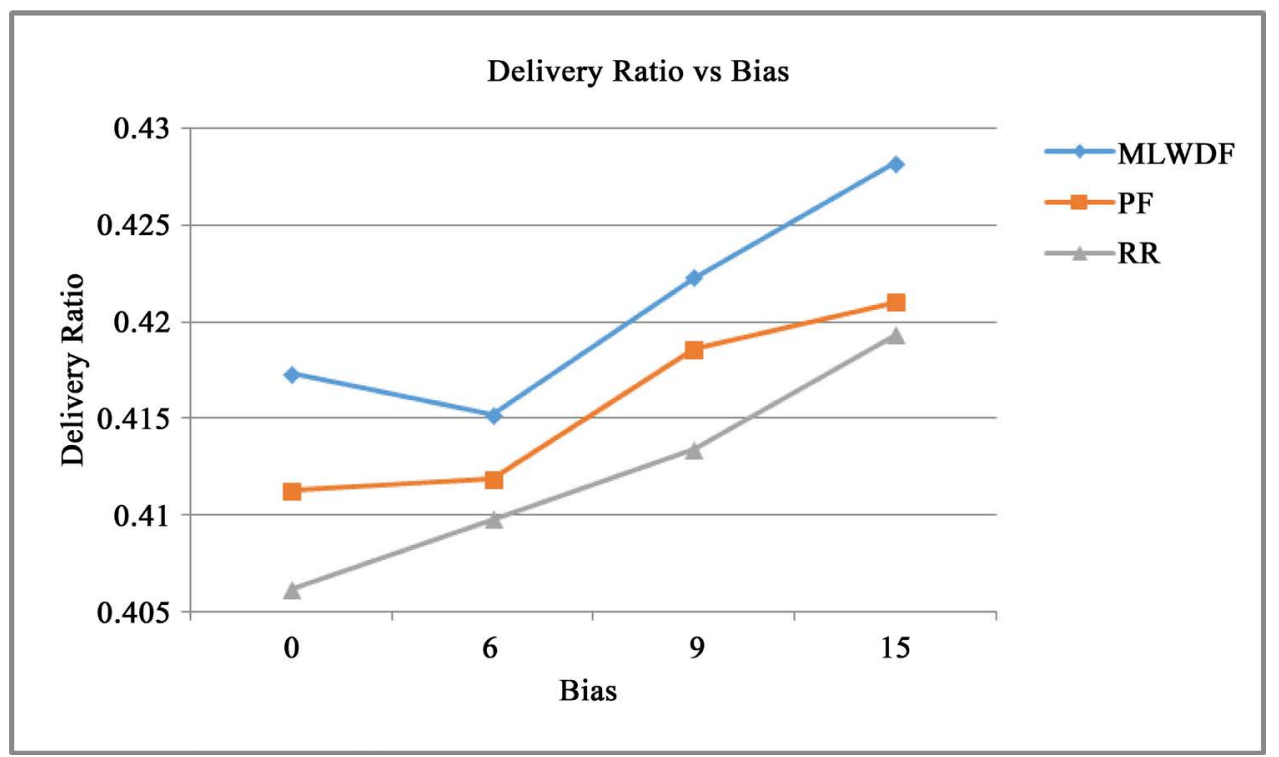

Figure 8. Packet delivery ratio performance comparison for various bias values using MLWDF, PF and RR schedulers.

mance with the additional QoS and delay metric compared to the PF scheduler. The RR scheduler performs poor for the similar reasons explained for the results in Figure 7.

\section{Conclusion}

The interference suppression capability of the MMSE and eMMSE receiver with a single dominant interference is analyzed, and their BER performance is studied. The link level performance of both receivers is compared with single eNB antenna and various UE antenna configurations. The performance of both receivers can be improved with increasing number of antennas at the UE. Moreover, it is found that both the receivers are able to completely suppress the interference when the number of antennas at the UE is sufficiently large. The system level performance of a system having one eNB and two pico eNB using MLWDF, PF, and RR schedulers is carried out by allocating the resources based on the post processing SINR of the eMMSE receiver. The throughput, average delay, and packet delivery ratio performances are obtained and compared for various biasing value of 3 , 
6, 9, and $15 \mathrm{~dB}$. It is found that the MLWDF scheduler achieves higher throughput with the least average packet delay and the best packet delivery ratio compared to the RR and PF schedulers. The next best performance is obtained for a PF scheduler, and RR scheduler achieves the worst performance for all the three performance metrics. The performance of these schedulers along with further eMMSE receivers that can handle more number of eNB interferences using higher number of UE antenna configurations could be further explored.

\section{References}

[1] Feng, M.J., Jiang, T., Chen, D. and Mao, S.W. (2014) Cooperative Small Cell Networks: High Capacity for Hotspots with Interference Mitigation. IEEE Wireless Communications, 21, 108-116. http://dx.doi.org/10.1109/MWC.2014.7000978

[2] Damnjanovic, A., et al. (2011) A Survey on 3GPP Heterogeneous Networks. IEEE Wireless Communications, 18, 10-21. http://dx.doi.org/10.1109/MWC.2011.5876496

[3] Ghosh, A., Ratasuk, R., Mondal, B., Mangalvedhe, N. and Thomas, T. (2010) LTE-Advanced: Next Generation Wireless Broadband Technology. IEEE Wireless Communications, 17, 10-22. http://dx.doi.org/10.1109/MWC.2010.5490974

[4] Pauli, V., Naranjo, J.D. and Seidel, E. (2010) Heterogeneous LTE Networks and Inter-Cell Interference Coordination. Nomor Research GmbH, Munich, 1-9.

[5] Gerhard, W., et al. (2015) 5G Now Final MAC/Networking Concepts. 5GNow_D4.2_Final. http://www.5gnow.eu/wp-content/uploads/2015/04/5GNOW_D4.2_final1.pdf

[6] Supratim, D., Pantelis, M., Jerzy, M. and James, P.S. (2014) Algorithms for Enhanced Inter-Cell Interference Coordination (eICIC) in LTE HetNets. IEEE/ACM Transactions on Networking, 22, 137-150.

[7] Andrews, J.G., Choi, W. and Heath Jr., R.W. (2007) Overcoming Interference in Spatial Multiplexing MIMO Cellular Networks. IEEE Wireless Communications, 14, 95-104. http://dx.doi.org/10.1109/MWC.2007.4407232

[8] Balint, C., Budura, G. and Marza, E. (2010) Scheduling Techniques Evaluation in LTE systems with Mixed Data Traffic. Proceedings of the 9th International Symposium on Electronics and Telecommunications, Timisoara, 11-12 November 2010, 221-224.

[9] Gomez, K., Goratti, L., Granelli, F. and Rasheed, T. (2014) A Comparative Study of Scheduling Disciplines in 5G Systems for Emerging Communications. Proceedings of the 1st International Conference on $5 G$ for Ubiquitous Connectivity, Akaslompolo, December 2014, 1-6.

[10] Capozzi, F., Piro, G., Grieco, L.A., Boggia, J., and Camarda, P. (2013) Downlink Packet Scheduling in LTE Cellular Networks: Key Design Issues and a Survey. IEEE Communications Surveys \& Tutorials, 15, 678-700. http://dx.doi.org/10.1109/SURV.2012.060912.00100

[11] 3rd Generation Partnership Project, Technical Specification Group, Radio Access Network (2013-02) Evolved Universal Terrestrial Radio Access (E-UTRA), Physical Channels and Modulation. Release 10, 3GPP TS 36.211 V10.7.0. http://www.3gpp.org/ftp/Specs/archive/36_series/36.211/36211-a70.zip

[12] Ikuno, J.C., Wrulich, M. and Rupp, M. (2010) System Level Simulation of LTE Networks. Proceedings of the 71st IEEE Vehicular Technology Conference, Taipei, 16-19 May 2010, 1-5.

[13] 3rd Generation Partnership Project, Technical Specification Group, Radio Access Network (2011-03) Spatial Channel Model for Multiple Input Multiple Output (MIMO) Simulations. Release 10, 3GPP TR 25.996.

http://www.3gpp.org/ftp/Specs/archive/36_series/36.211/36211-a70.zip

[14] Tetsushi, A. and Gerhard, B. (2010) Effective SINR Computation for Maximum Likelihood Detector in MIMO Spatial Multiplexing System. Proceedings of the IEEE Global Telecommunication Conference, Honolulu, 30 November-4 December 2009, 1-5.

[15] Eraslan, E., Daneshrad, B. and Lou, C.-Y. (2012) Performance Indicator for MIMO MMSE Receivers in the Presence of Channel Estimation Errors. IEEE Wireless Communication Letters, 2, 211-214. http://dx.doi.org/10.1109/WCL.2013.012513.120824

[16] Bai, Z.J., et al. (2011) On the Equivalence of MMSE and IRC Receiver in MU-MIMO Systems. IEEE Communication Letters, 15, 1288-1291. http://dx.doi.org/10.1109/LCOMM.2011.093011.110826

[17] Tavares, F.M.L., Berardinelli, G., Mahmood, N.H., Sorensen, T.B. and Mogensen, P. (2014) On the Impact of Receiver Imperfections on the MMSE-IRC Receiver Performance in 5G Networks. Proceedings of the 79th IEEE Vehicular Technology Conference, Seoul, 18-21 May 2014, 1-6.

[18] Tang, T.W., Doostnejad, R. and Lim, T.J. (2010) Mean Mutual Information Per Coded Bit Based Precoding in MIMOOFDM Systems. Proceedings of the 72nd IEEE Vehicular Technology Conference, Ottawa, 6-9 September 2010, 1-5. 


\section{Submit or recommend next manuscript to SCIRP and we will provide best service for you:}

Accepting pre-submission inquiries through Email, Facebook, LinkedIn, Twitter, etc.

A wide selection of journals (inclusive of 9 subjects, more than 200 journals)

Providing 24-hour high-quality service

User-friendly online submission system

Fair and swift peer-review system

Efficient typesetting and proofreading procedure

Display of the result of downloads and visits, as well as the number of cited articles

Maximum dissemination of your research work

Submit your manuscript at: http://papersubmission.scirp.org/ 